\title{
The use of relationship matrices to avoid inbreeding in small horse populations
}

\author{
O. VANGEN \\ Department of Animal Genetics and Breeding \\ Agricultural University of Norway, $\stackrel{\circ}{s-N L H, \text { Norway }}$
}

Computer programs have made it possible to calculate exact inbreeding for all animals in a population through large numerator relationship matrices. As all off-diagonal elements of such a matrix can be calculated as functions of diagonal elements, the capacity of inbreeding and relationship calculations has been largely increased and can easily handle 40000 animals.

This method has been used in the three native Norwegian horse breeds, Dele-horse, Fjord-horse and Nordland-horse, to calculate complete inbreeding coefficients for 37641 , 18882 and 218 animals in the three breeds, respectively. The results are given in the text.

The practical applications of the knowledge of complete inbreeding and relationship in a breed is discussed in relation to the possibilities of to some extent avoiding inbreeding in small horse populations. As the selection criteria often are not well defined, some of the selection policy could easily be attained to the inbreeding problem.

\section{Breeding problems in the Friesian horse}

\author{
W. van der HOLST \\ Vakgroep Verloskunde, K.I. en voorplanting, Afdeling K.I., Fac. Diergeneeskunde \\ Yalelaan 7, De Uithof, Utrecht, Netheriands
}

The Friesian horse has been bred pure for a hundred years and no fresh blood has ever been introduced. This stud was during the last 30 years threatened by many setbacks. In 1971 the use of A.I. led to the discovery of breeding problems in mares and stallions and then on inventory of these problems was begun.

Dwarf-foalism was estimated in about $5-10 \%$ in the offspring of carriers. Carriers were never selected against as dwarffoalism is not considered a serious threat to the breed; most of these carriers have more favourable qualities.

Since 1972 it is compulsory for young stallions to have a sperm examination before they are admitted to the stud.

The inbreeding coefficient of disqualified stallions during the period 1972 to 1977 was high $(0.332 ; \mathrm{n}=23)$.

During the period 1977 to 1981 we found for the inbreeding coefficient of the same category stallions $0.268(n=81)$.

The figures for the first, repectively secound group of approved stallions were 0.212 and 0.158 .

Selection on sperm quality and on degree of inbreeding did not fail to produce results in the second group of stallions.

Crytorchism on one or both sides was found significantly more often in stallions from strongly inbred bloodlines up till $50 \%$ of the 2,5 year old stallions.

In a dozen of cases aplasia or hypoplasia of parts of the sexual organs of stallions was found (0.278). 
A large number of mares $(35 \%)$ have a retained placenta for more than 6 hours. Preliminary calculations lead to the conclusion that the more closely related the parents of a foal are, the more frequently these problems occur.

Certain combinations of bloodlines showing a $30 \%$ increase of the probability of underdeveloped ovaries in the mares.

Suggestions are being made for a breeding programme for the Friesian horse.

\title{
Genetic parameters of selection criteria in horses. \\ The effects of different calculative methods on the heritability estimates of racing ability of Thoroughbred horses
}

\author{
I. BODÓ and Elisabeth TAKĀCS \\ Department of Animal Husbandry, University of Veterinary Science \\ Budapest, 7 P.O.B. 2, Hungary 1400
}

Heritabilities of handicap weights of Thoroughbred horses were calculated using different mathematical methods. Twenty $\mathrm{h}^{2}$-values were obtained wich were in a range from 0 to 0.60 . Calculative methods exert a systematic influence on heritability estimates according to the structure of population, therefore his fact must be taken into consideration when evaluating the figures of such calculations.

SEsSION V b

AMÉLIORATION DES BOVINS LAITIERS

\section{Testing of dairy sires at different production levels}

\author{
M.K. AHMED *, W.G. HILL *, C.J.M. HINKS ** and R. THOMPSON *** \\ * Institute of Animal Genetics, Edinburgh EH9 $3 J N$, Scotland \\ ** ARC Animal Breeding Research Organisation, Edinburgh EH9 3JQ, Scotland \\ *** ARC Unit of Statistics, Edinburgh EH9 $3 J Z$
}

Herds were divided into two groups according to their mean heifer milk yield. Analyses of variance on heifer yields were conducted within levels on 9775 daughters of 225 unproven bulls, using 12610 daughters of widely used tested sires to generate additional connections. For milk, fat and protein yield greater heritabilities were obtained in the high than in the low level group of herds, but there was little difference for fat or protein percentage. Genetic correlations between performance in the two levels were close to one for all traits. 